behavioural examples rather than using well-chosen examples to develop and illustrate the experimental methodology and the principles underlying the analytical procedures and interpretations. It is difficult to believe that more appropriate examples could not have been found or that they could not have been used more effectively to illustrate the principles of experimental design, model building, hypothesis testing, interpretation and prediction. These are, however, relatively minor deficiencies compared with the advantages of having a concise, up to date account of the subject.

J. L. JINKS

\section{Deformed embryos}

Environment and Birth Defects. By James G. Wilson. Pp. xiv +305 . (Environmental Studies: an Interdisciplinary Monograph Series.) (Academic: New York and London, December 1973.) $£ 9.10$.

THOugh mortality at all ages in the period of childhood and adolescence has fallen dramatically during the past few decades mortality from congenital defects has shown little change. The relative importance of congenital defects has increased and continues to increase. This applies both to mortality and morbidity. Knowledge on the aetiology and underlying pathogenesis of congenital defects is very limited and much of the information available is widely dispersed in the literature. A book which brings together and unifies much of the existing information on teratology-defined as the adverse effect of environment on developing systems - is to be welcomed and fills a gap in the medical and biological literature.

The book begins by identifying the broad issues in teratology including the basis on which the conceptus becomes susceptible to adverse effects; the disturbance of cellular tissue and developmental processes which constitute the basic pathology of teratology; the importance of accessibility of an agent to the conceptus in determining possible teratogenic effects; dose effects; and the possible range of manifestations of deviant development. The causes of developmental anomalies are discussed in terms of genetic influences, chromosomal aberrations, radiation, chemicals and drugs, dietary imbalance, infections, respiratory gas levels, extremes of temperature, metabolic or endocrine imbalance, physical trauma, mechanical factors, placental factors, antibodies and combined effects. A review of the various drugs known or suspected to cause congenital malformations (not entirely complete) is relevant to clinical pharmacology and it touches on the possible teratogenic effects of environmental chemicals used in industry and in agriculture.

The book then goes on to discuss the means by which developmental defects occur-the mechanisms of teratogenesis -under the headings of mutation; chromosomal non-disjunction and breaks; mitotic interference; altered nucleic acid integrity or function; deficiency of precursors, substrate and enzymes; altered energy sources; enzyme inhibition; osmolar imbalance; changed membrane characteristics; excessive cell death; reduced cell proliferation; disturbed interaction between cells; and reduced migratory movement of cells. Figures are given for the general incidence of developmental defects in man based on differing assessments made on embryos, stillbirths, neonatal deaths and later deaths. The effect of teraiogenic processes is classified into four categories, namely, intrauterine death, malformation, growth retardation or functional defect.

There is a useful account of the early developmental stages of the human ovary and foetus with pictorial representation of 23 different stages from fertilisation to the 53rd day of gestation, and a discussion on the concept of critical periods of susceptibility.

Further chapters deal more specifically with the access of teratogenic agents tc the foetus and the foetal defence mechanism against them, and with the means of detection of teratogenic effects in man including recognition based on observation of clusters of cases, epidemiological surveys and surveys of high risk populations.

The last quarter of the book deals largely wth the contribution of animal experiments to the assessment of human teratogenic risk and to the problems which arise from such experiments. The latter include the differing effeci caused by the timing of any insult, dose effect in respect of quantity of teratogen and duration of administration, the masking of teratogenesis by embryolethal effec $i$ and standards of recognjtion of defects. There is a section on the various animals which can be used in teratology testing. The author puts forward a suggested scheme of teratogenic testing based on the graded use of animals of different availability and cosî. Lastly there are special sections on appropriate literature relative to a number of individual animals and there is a valuable general bibliography.

This book ranges over the field of teratogenic response to the environment as revealed by animals and human studies. It concentrates primarily on the broader biological issues of teratogenesis approaching the subject from a morphological rather than a biochemical standpoint. The principles of teratological testing are discussed and the author's own recommendations regarding his scheme for animal testing of suspect drugs provides a special component to the discourse. The book is clearly and concisely written and will prove a very valuable source of information to a wide range of those who are concerned with the environment and its possible effect on human development. These would include environmental scientists, hygienists, developmental biologists, obstetricians and paediatricians, toxicologists and pharmacologists. JOHN O. FORFAR

\section{Mathematical brainwaves}

Automation of Clinical Electroencephalography. Edited by Peter Kellaway and Ingemar Petersén. Pp. viii +318. (Raven: New York; North-Holland: Amsterdam, 1973.) \$22.70; Dfl.59.

THIS book provides an excellent guide to the quantitative methods of analysis currently being employed in EEG research. In the opening chapters, the editors draw attention to the major problem involved, namely that of defining the standard method of visual analysis in mathematical terms. For the non-clinician, a comprehensive description of standard EEG analysis is included, together with examples of typical wave forms and commoner abnormalities.

The case is presented for regional centres for automatic EEG analysis, so that research methods described can be widely applied to clinical practice. To implement this programme, a telemetry system capable of carrying at least eight channels of information would be required, a suitable system operating by public telephone being described later in the book. Continuing this theme, methods are describe! enabling multichannel EEG data to be presented in a simplified form on a single page, either by bar graphs for the principal frequency bands (delta. theta, alpha, beta), or by compressed spectral array, neither technique requiring as experienced an interpreter as conventional paper records. Extensive data reduction is involved, and although the two methods are complementary, they are suitable only as screening tests, and not as replacements for conventional EEG techniques. The method and application of Fast Fourier Transform are considered, and among many other topics included are pattern recognition techniques and EEG analysis in sleep.

The book is well presented and clearly illustrated throughout, and should prove a valuable reference work for those with interests in this expanding field.

L. M. BRaNCH 